\title{
Prospects for the study of baryon-rich matter at new facilities
}

\author{
Volker Friese* \\ GSI Helmholtzzentrum für Schwerionenforschung mbH, Darmstadt, Germany \\ E-mail: v.friesedgsi.de
}

In the next decade, several new accelerator facilities will come into operation, which will offer unprecedented opportunities for the experimental investigation of strongly interacting matter at high net-baryon densities as created in nucleus-nucleus collisions at moderate energies. The new experiments will complement existing and running experiments in this field and enhance the physics reach with increased rate capabilities. In this article, we give an overview of the facilities and experiments currently under construction, compare their capabilities, and briefly discuss the physics prospects opening up with the data from these experiments.

Corfu Summer Institute 2018 "School and Workshops on Elementary Particle Physics and Gravity" (CORFU2018)

31 August - 28 September, 2018

Corfu, Greece

\footnotetext{
${ }^{*}$ Speaker.
} 


\section{Introduction}

The terrestrial tool to study the properties of strongly interacting matter off ground state are to collide heavy nuclei and extract information on the created medium from the emitted reaction products. Experiments of this kind have been performed over the past four decades at ever increasing energies as becoming available with new accelerators: Bevalac, SIS-18, AGS, SPS, RHIC, and LHC. Today's evidence is that at the highest energies studied so far, the matter created in nuclear collisions in in a deconfined and chirally symmetric state - in line with lattice QCD calculations predicting a chiral cross-over at a critical temperature of $T_{c}=156 \mathrm{GeV}$ at vanishing baryo-chemical potential [1].

Theoretical first-principle access to QCD matter at finite net-baryon density is limited. QCDinspired effective models, however, predict a rich structure of the QCD phase diagram, including first-order deconfinement and chiral phase transitions and a critical end-point [2]. The experimental verification of such structures is the prime aim of the heavy-ion programmes at moderate collision energies, making use of the fact that by variation of the collision energy, different regions of the phase diagram can be probed. A key feature of the search for the QCD landmarks is thus to study the energy dependence of observables believed to be sensitive to deconfinement, chiral symmetry or critical behaviour. Non-monotoniticities in such excitation functions are the most promising signals for phase transition and critical point. The first to execute such an "energy scan" was the NA49 experiment at the CERN-SPS (1999-2002), followed by the beam energy scan of the STAR experiment at BNL-RHIC (BES-1, 2010-2012).

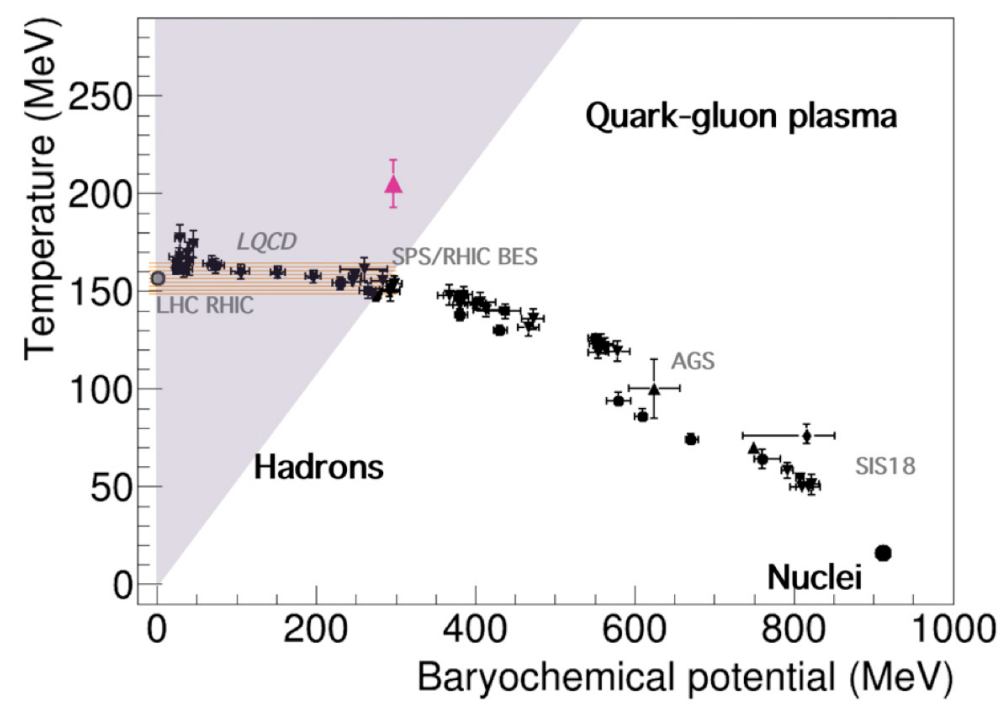

Figure 1: Freeze-out points in baryo-chemical potential and temperature for heavy-ion collisions at different collision energies, obtained from fits of the measured particle abundances with the Statistical Hadron Gas Model (taken from [3]). The shaded area shows the region accessible with current lattice-QCD calculations.

The data collected so far mostly covered bulk observables and allowed to characterise the thermal properties of the medium (Fig. 1). The key questions, however, remain to be answered: what is 
the equation-of-state of nuclear matter at high baryon densities; is there a first-order deconfinement and/or chiral phase transition; is there a critical point, and if yes, where?

These question will be addressed in the near future by experiments at existing accelerator facilities. The BES-2 programme of the STAR experiment will renew the efforts of BES- 1 with an upgraded detector and increased rate capability in the years 2019 and 2020 [4]. It will also extend the accessible energy range by measuring in fixed-target mode with an internal target in the beam halo. The NA61 detector - a successor of NA49 - is proposed for a run campaign at SPS from 2022 on, also being upgraded with a Micro-Vertex Detector and a new data acquisition allowing to measure at higher interaction rates [5]. In discussion is the NA60+ experiment for di-muon measurements at the SPS, its detector concept being based on the successful NA60 experiment [6].

In addition to these activities, several new accelerator facilities are currently under construction or proposed. The aim of the experimental programmes at these facilities is to overcome the limitations of existing experiments, in particular in energy range and rate capability, by next-generation experiments making use of state-of-the-art detector technology, read-out electronics and data acquisition. The focus of the experimental projects is high rate capability, allowing to systematically study also rare observables. In the following section, we give an overview of the new facilities and experiments. Their operational conditions will be compared in section 3; a quick look into the physics prospects opened by the new programmes will be given in section 4 .

\section{New facilities and experiments}

\subsection{NICA}

The Nuclotron-based Ion Collider Facility (NICA) [7] is one of the six Russian megascience projects and is being realised at the Joint Institutes of Nuclear Research in Dubna, Russia. The planned accelerator complex is shown in Fig. 2, left. It is based on the existing Nuclotron, which is being upgraded to higher beam intensity and quality. A new Linac will deliver heavy ions into a Booster ring, where ions are accumulated and pre-accelerated before being injected into the
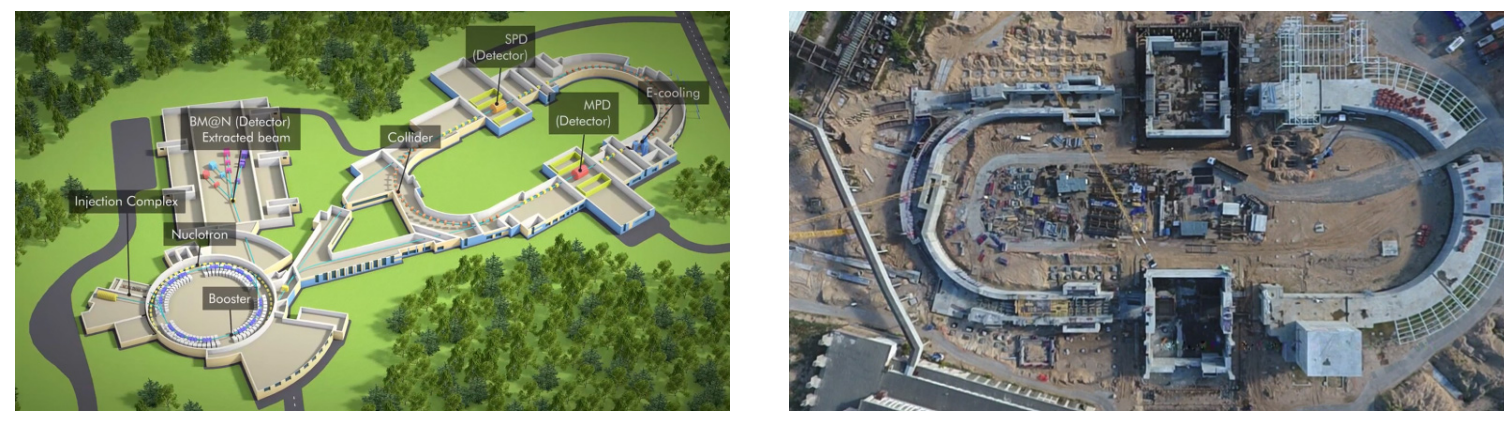

Figure 2: Left: Layout of the NICA complex [8]. Below the existing Nuclotron, the new Booster ring is installed. Beams are extracted from the Nuclotron to fixed-target experiments in a new experimental area or injected into the two collider rings (under construction). At the two intersection points, the MPD and the SPD detectors will perform experiments. Right: aerial view of the NICA construction site in summer 2018 [10]. 
Nuclotron. The Nuclotron, with a rigidity of $45 \mathrm{Tm}$, accelerates ions up to $4.5 \mathrm{GeV} / \mathrm{u}$ beam kinetic energy. These beams can be slowly extracted for external, fixed-target experiments or injected into two superconducting collider rings with two interaction points. At its top energy $\left(\sqrt{s_{\mathrm{NN}}}=11 \mathrm{GeV}\right)$, the collider aims at a luminosity of $10^{27} \mathrm{~cm}^{-2} \mathrm{~s}^{-1}$.

The construction of the facility is under way (Fig. 2, right). Both the new injection complex and the Booster ring are commissioned in 2018. Civil construction for the collider ring and its experimental halls are expected to be finished in 2019, and first collisions at NICA are foreseen in 2021.

Heavy-ion physics with extracted beams from the Nuclotron will be performed by the BM@N detector (Fig. 3). It is a fixed-target spectrometer covering a large acceptance in the forward rapidity hemisphere. A large-aperture dipole magnet houses a system of radiation-hard tracking devices (GEM detectors). Hadron identification is achieved by the measurement of time-of-flight, which is connected to the spectrometer by an outer tracking system composed of Cathode Strip Chambers and multi-wire drift chambers. A forward calorimeter serves for event characterisation. BM@N will investigate heavy-ion collisions at beam kinetic energies from 1 to $4.5 \mathrm{GeV} / \mathrm{u}$ at an interaction rate of up to $10 \mathrm{kHz}$. It will be able to identify hadrons by time-of-flight and hyperons and hyper-nuclei by their decay topology. Its physics focus is the investigation of the nuclear equationof-state at high net-baryon densities through the measurement of the collective flow of identified hadrons and the excitation function of hyperons. The experiment was already commissioned with light ion beams (Ar, Kr) from the Nuclotron in March 2018 [9]. Experiments with heavy beams are expected for 2020. From 2021 on, an upgrade of the tracking system with Silicon detectors is foreseen, allowing to increase the interaction rate to $50 \mathrm{kHz}$.
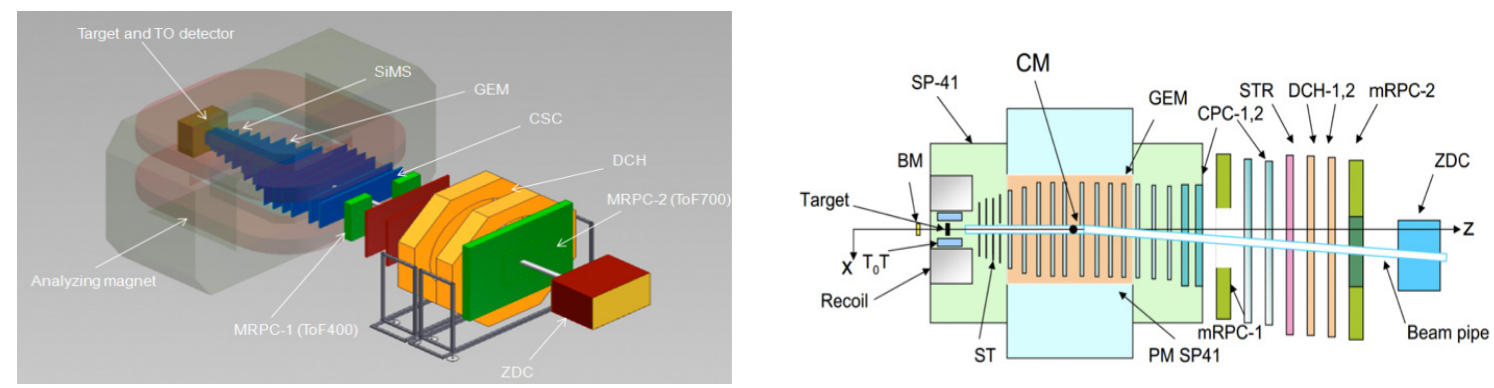

Figure 3: The BM@N experimental setup [10]. Left: experiment layout; right: schematic view of the detector systems. BM: beam monitor; ST silicon tracker; GEM: gas electron multiplier detectors; CPC: Cathode Strip Chambers; DCH: drift chambers; mRPC: multi-gap resistive place chambers (TOF); ZCD: zero-degree calorimeter.

The Multi-Purpose Detector (MPD) is being designed to perform experiments at one of the interaction points of the new NICA collider rings. Figure 4 shows the barrel-like detector setup typical for collider experiments. The Time-Projection Chamber reconstructs tracks in the superconducting magnet in a pseudo-rapidity range of $|\eta|<1.2$. Hadron and electron identification is achieved by the measurement of time-of-flight and the specific energy loss in the TPC. An electromagnetic calorimeter allows the measurement of charge-neutral probes. MPD will start experiments in 2021. In a second stage (2023), the experiment will be upgraded with endcap systems 
(TOF and ECAL), increasing the acceptance in rapidity space, and an inner tracking system for the detection of decays of charmed hadrons. The energy range of MPD is $\sqrt{{ }^{s} \mathrm{NN}}=4-11 \mathrm{GeV}$; the maximal interaction rate at top energy is $7 \mathrm{kHz}$.
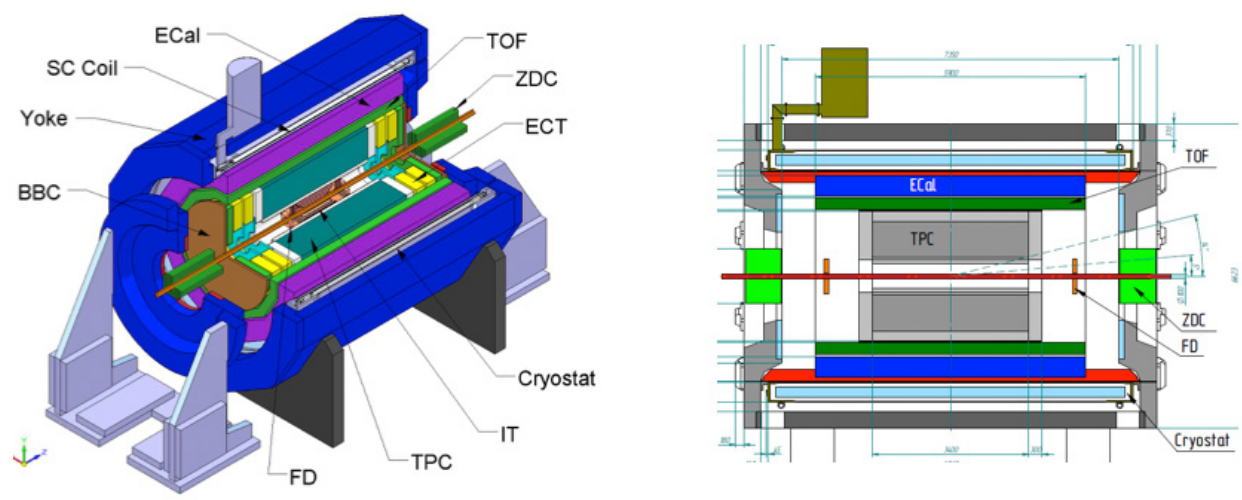

Figure 4: The MPD experiment at NICA [10]. The collision point in the centre of the setup. TPC: timeprojection chamber; TOF: time-of-flight detector; ECal: electro-magnetic calorimeter; FD: forward detector; BBC: beam-beam counter; ZDC: zero-degree calorimeter.

\subsection{FAIR}

The Facility for Anti-Proton and Ion Research (FAIR) is currently being constructed in Darmstadt, Germany, adjacent to the GSI research centre [11]. FAIR will comprise a complex system of accelerators, storage and cooler rings, and experiment beam lines, the core of which being the SIS-100 synchrotron with a bending power of $100 \mathrm{Tm}$. The facility is designed to serve a variety of physics programmes, ranging from nuclear structure physics with rare-isotope beams, plasma physics with highly bunched ion beams, atomic physics with ion and anti-proton beams, hadron structure physics with anti-proton beams, and nuclear matter physics with heavy-ion beams. FAIR is currently the largest European project in fundamental research, comprising an area of $200,000 \mathrm{~m}^{2}$, half of which covered by buildings with a total volume of about one million $\mathrm{m}^{3}$, and about $3.2 \mathrm{~km}$ of beam lines (1.1 km for the synchrotron). The facility is an international project with eight shareholder countries (Germany, Finland, France, India, Poland, Portugal, Slovenia, Sweden, Russia and the United Kingdom). About 2,500 scientific users are currently preparing the experiments.

A schematic view of the full facility is shown in Fig. 5 together with an artistic view of the site after completion of constructions. The construction of the FAIR site started in July 2017 with excavations for the beam lines and trench sheeting. In July 2018, the shell construction has started (Fig. 6). According to the current planning, the buildings will be completed by 2022, and completion of the full facility in its start version and the beginning of the operations is foreseen for 2025. In a later stage (phase 2), a second booster synchrotron (SIS-300) will enlarge the maximal beam energy with a bending power of $300 \mathrm{Tm}$ (or more), and experimental facilities for research with low-energy anti-protons will be added. The ring tunnel building already foresees the space for the installation of the second accelerator. Apart from extending the beam energy range, it 

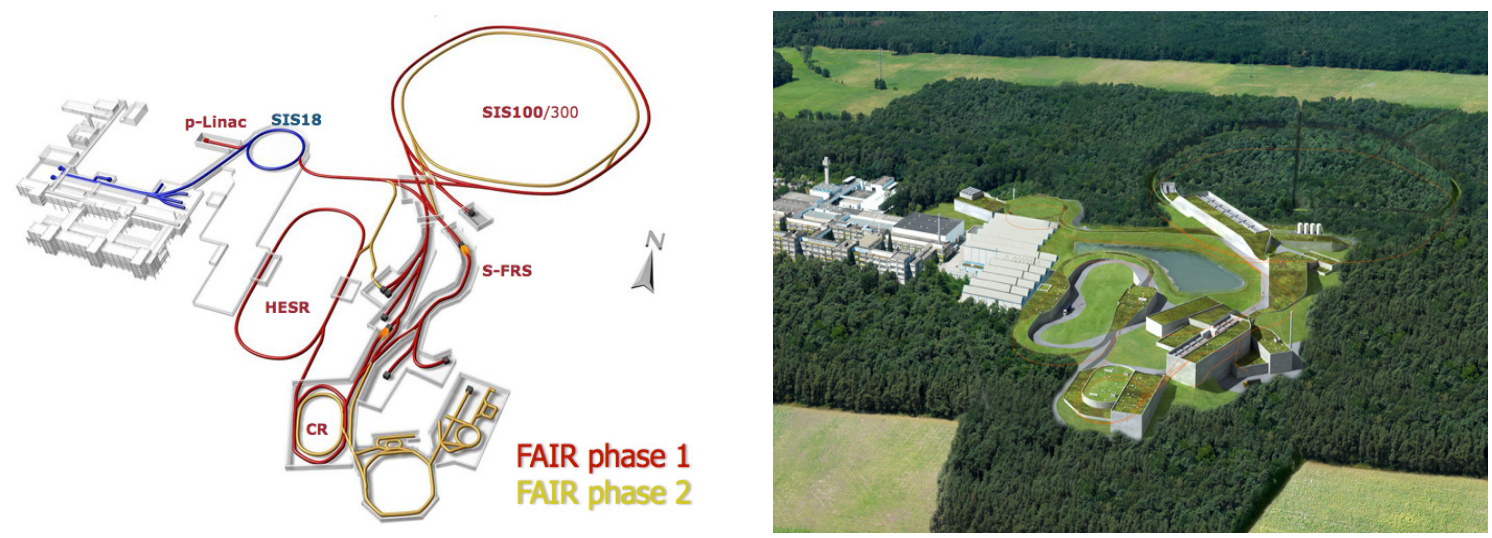

Figure 5: The FAIR facility [12]. Left: schematic view of the accelerator complex. The existing accelerators of GSI (Unilac, SIS-18, shown in blue) serve as injectors to the SIS-100 synchrotron. S-FRS: Super Fragment Separator; HESR: High-Energy Storage Ring for anti-protons; CR: Cooler Ring for anti-protons. The beam lines shown in yellow will be realised in phase 2. Right: artistic view of the completed research facility.

will significantly enhance the possibilities for parallel operations of the facility, serving different physics programmes at a time and thus accelerating the physics output.

For the nuclear collision programme, SIS-100 will deliver slowly extracted, fully stripped heavy ions up to a beam kinetic energy of $11 \mathrm{GeV} / \mathrm{u}$ with high intensity of above $10^{10}$ ions per second. For symmetric nuclei, the maximal kinetic energy is $14 \mathrm{GeV} / \mathrm{u}$; proton beams will be available up to $29 \mathrm{GeV}$. These beams will be used by the Compressed Baryonic Matter (CBM) experiment, which is being prepared for taking first data in 2025 [14, 15]. CBM is a fixed-target spectrometer with large acceptance in the forward rapidity hemisphere. Its detector setup, depicted in Fig. 7, comprises systems for tracking in the dipole magnetic field (Silicon Tracking System, STS), hadron identification by time-of-flight, and electron identification (Ring-Imaging Cherenkov

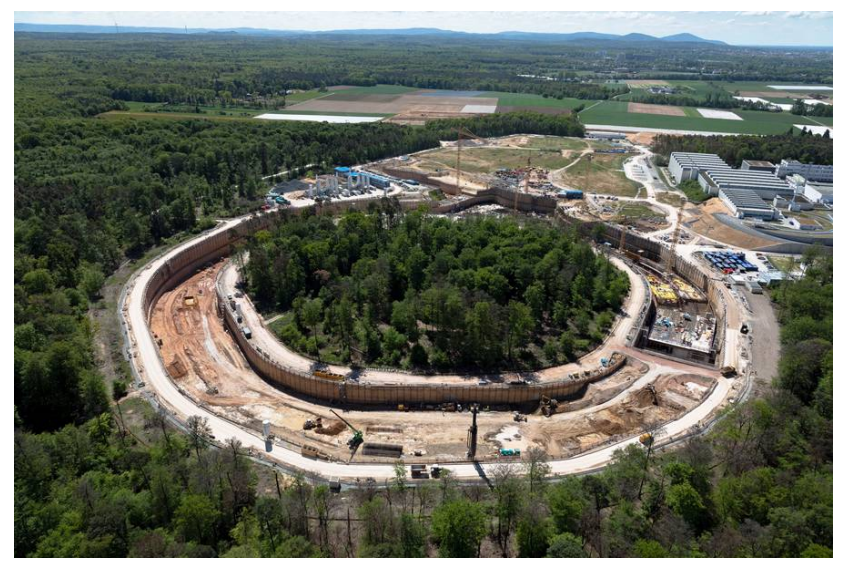

Figure 6: The FAIR construction site in May 2019, seen from north [13]. The shell construction for the synchrotron tunnel and the CBM cave is in progress. 
detector, RICH, and Transition-Radiation Detector, TRD). Alternating to the RICH, a muon detection system (MUCH), realised by a set of tracking detectors placed in between hadron absorbers, will be used to identify muons. A Micro-Vertex Detector (MVD) enables the detection of displaced vertices of charmed-particle decays and the detection of gamma conversions to reduce the background in di-electron studies. CBM aims at a rate capability of up to $10^{7}$ collisions per second, which would be more than two orders of magnitude higher than any existing heavy-ion experiment. This ambitious goal will be reached with fast detectors, trigger-less read-out electronics and a streaming data acquisition, where real-time event building and selection will be performed exclusively in software [16]. By design, most of the CBM detectors are also suitable for nuclear collisions at higher energies as will become available in FAIR phase 2 with the additional booster synchrotron.

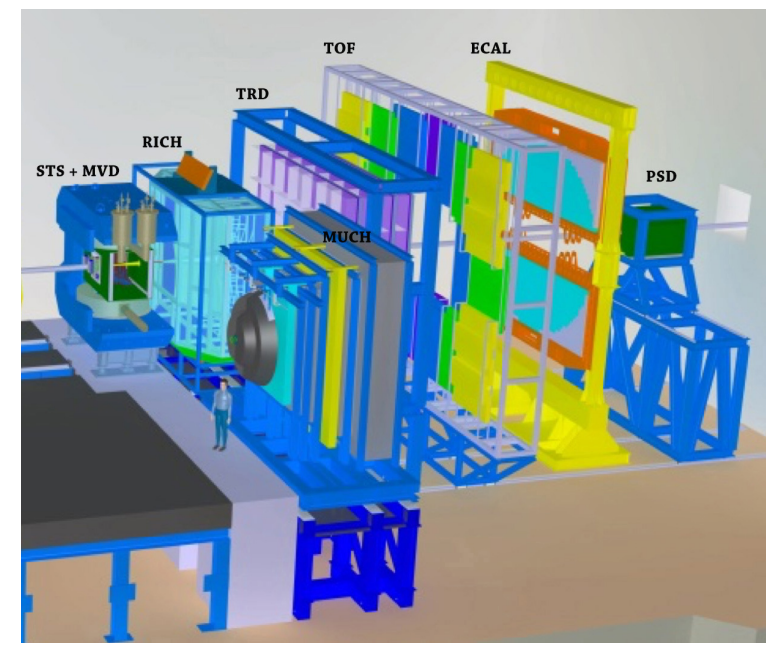

Figure 7: The CBM experimental setup. The beam enters from the left. Within the dipole magnet, the target, the Silicon Tracking System (STS) and the Micro-Vertex Detector (MVD) are placed. RICH: RingImaging Cherenkov detector; TRD: Transition-Radiation Detector; TOF: Time-Of-Flight detector; ECAL: Electromagnetic Calorimeter; PSD: Projectile Spectator Detector.

In addition to CBM, the HADES experiment, currently taking data at the GSI SIS-18, will also measure nuclear collisions with beams from the SIS-100. To this behalf, the detector will be moved to the CBM experimental hall at the new facility. Although limited for heavy-ions by granularity to low beam kinetic energies (up to $4 \mathrm{GeV} / \mathrm{u}$ for medium-sized nuclei), the HADES backward acceptance makes it a well-suited tool for reference measurements in proton-induced reactions, which are indispensable for the physics interpretation of experimental results in heavyion collisions. 


\subsection{HIAF}

The Heavy-Ion Accelerator Facility (HIAF) in Huizhou, China, is one of the large-scale facilities in China to boost basic science within the $12^{\text {th }} 5$-years-plan [17]. First proposed in 2009, the project was approved in 2015, and construction has started at the end of 2018. The start of operation is planned for 2024 . The facility will provide primary and secondary ion beams for nuclear structure physics, nuclear matter physics, plasma physics, and applied physics. A schematic view of the facility is shown in Fig. 8, left. It comprises a Linac, a Booster ring (BRing) and a Spectrometer Ring (SRing). The BRing, with a rigidity of $34 \mathrm{Tm}$, will deliver extracted heavy-ion beams with a beam kinetic energy of up to $1.2 \mathrm{GeV} / \mathrm{u}$ and very high intensity (up to $10^{11}$ particles per pulse) for external experiments. Plans for a later additional booster ring would allow to extend the energy range to $3.8 \mathrm{GeV} / \mathrm{u}$.
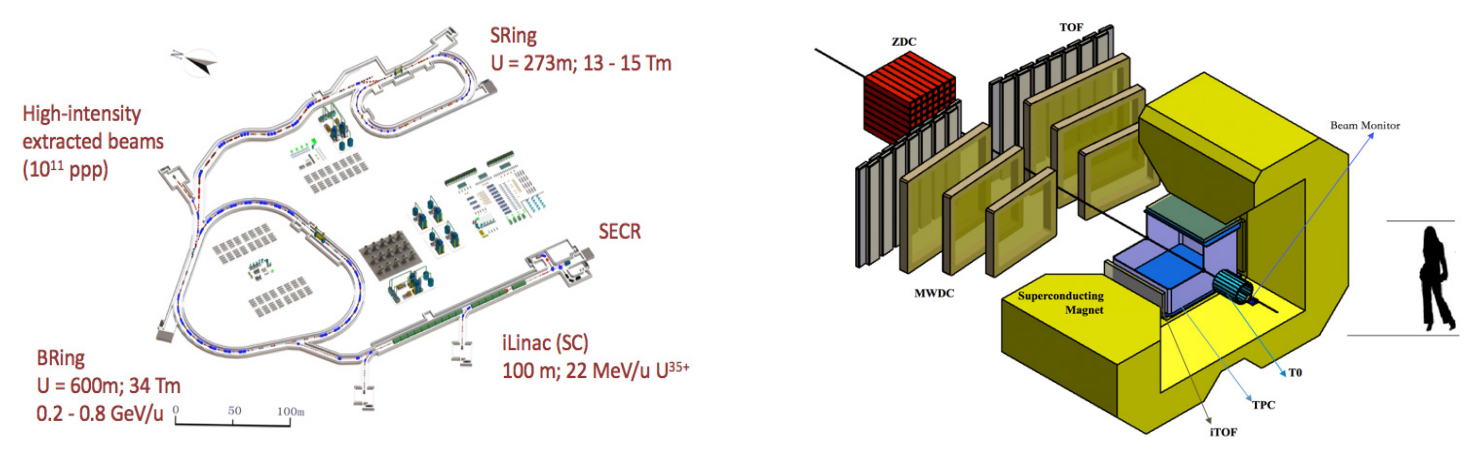

Figure 8: Left: Schematic view of the HIAF facility [18]. SECR: Superconducting Electron Cyclotron Resonance ion source; iLINAC: superconducting linear accelerator; BRing: high-intensity synchrotron Booster Ring; SRing: high-precision snchrotron Spectrometer Ring. Right: planned setup of the CEE experiment at HIAF [19]. TO: start detector; TPC: time-projection chamber; TOF / iTOF: (inner) time-of-flight detector; MWDC: multi-wire drift chambers; ZDC: zero-degree calorimeter.

Research on QCD matter at HIAF will be conducted by the planned CEE experiment. CEE is being designed as fixed-target, forward spectrometer, tracking particles in a dipole magnetic field by a time-projection chamber and identifying protons and pions by means of time-of-flight. The collision centrality is determined by a zero-degree calorimeter. Fig. 8 (right) depicts the experimental setup. CEE targets for collision rates up to $10^{5}$ per second, being limited by the drift time in the TPC. As for CBM, the readout is planned to be free-streaming. Physicswise, CEE focuses on the measurement of event-by-event proton fluctuations, directed flow, and symmetry energy through proton flow and the $\pi^{+} / \pi^{-}$ratio.

\subsection{J-PARC-HI}

The Japan Proton Accelerator Research Complex (J-PARC) currently provides high-intensity proton beams. It is proposed to extend the physics reach of the facility by the construction of a heavy-ion acceleration scheme [20], comprising a new heavy-ion Linac and a booster ring, connecting to the existing Rapid Cycling Synchrotron. When realised, the system would allow to perform nuclear collision experiments in fixed-target mode with beam kinetic energies from 1 to 
$19 \mathrm{GeV} / \mathrm{u}$. The anticipated beam intensities are extremely high ( $10^{11}$ ions per second) and would enable very high-rate experiments.

Several detector systems are being proposed for heavy-ion physics at J-PARC [21]. In a first stage, at moderate interaction rates, a Dipole Hadron Spectrometer with a time-projection chamber, a MRPC time-of-flight system and a neutron counter shall give access to hadronic probes. In a second stage, a muon tracker will replace the TPC to perform dilepton measurements at interaction rates of $10^{7} / \mathrm{s}$. The third stage addresses the measurement of hyper-nuclei at extreme interaction rates of up to $10^{8} / \mathrm{s}$. Here, the idea is to shield the detector from bulk particles by a sweeping magnet and a collimator, such that only beam fragments containing hyper-nuclei will enter the spectrometer.

In contrast to the facilities described before, which are already under construction, the J-PARC heavy-ion project is in the proposal stage, having recently been submitted to the 10-year plan of the Japanese Science Council for the decade starting 2020. When approved, a realisation of the accelerator and detector projects can be expected from the end of the decade on.

\section{Comparative view}

When judging the physics prospects arising with the new facilities outlined in the previous sections, the first criterion at hand is the accessible range of collision energy. A summary of the respective parameters is given in Table 1, showing that new experiments (CEE, BM@N, CBM, MPD) together cover complete the energy range $\sqrt{{ }_{\mathrm{NN}}}=1.8-11 \mathrm{GeV}$. In conjunction with the existing experiments (HADES, NA61, STAR), this means that the phase diagram of QCD can then be explored over the entire range, at baryo-chemical potentials from close to zero up to the highest values accessible in terrestrial experiments. Substantial overlap in collision energy coverage by the various experiments will allow to cross-check experimental results.

\begin{tabular}{|c|c|c|c|c|}
\hline Experiment & $\sqrt{{ }_{\mathrm{NN}}}[\mathrm{GeV}]$ & Mode & Max. interaction rate $\left[\mathrm{s}^{-1}\right]$ & Start \\
\hline \hline HADES (GSI) & $2.4-2.9$ & fixed target & $2 \cdot 10^{4}$ & 2012 \\
STAR (BES-II) & $3.0-19.6$ & collider + fixed target & $2 \cdot 10^{3}$ & 2019 \\
NA61 (CERN) & $4.9-17.3$ & fixed target & $10^{3}$ & 2022 \\
\hline \hline BM@N (NICA) & $2.0-3.5$ & fixed target & $5 \cdot 10^{4}$ & 2020 \\
MPD (NICA) & $2.7-11.0$ & collider & $7 \cdot 10^{3}$ & 2021 \\
CEE (HIAF & $1.8-2.7$ & fixed target & $10^{5}$ & 2023 \\
CBM (FAIR) & $2.7-5.0(8.5)$ & fixed target & $10^{7}$ & 2025 \\
\hline \hline J_PARC_HI & $2.0-6.2$ & fixed target & $10^{8}$ & $\mathrm{n} / \mathrm{a}$ \\
NA60+ (CERN) & $4.9-17.3$ & fixed target & $10^{7}$ & $\mathrm{n} / \mathrm{a}$ \\
\hline
\end{tabular}

Table 1: Operation properties of existing experiments, experiments in preparation, and proposed experiments for the investigation of the QCD phase diagram at high net-baryon densities. The maximal energy for CBM in brackets will only become available in FAIR phase-2.

The second important parameter to judge the physics potential of the experiments is the accessible interaction rate, in particular in view of the fact that most of the promising observables 
are quite statistics-demanding. A compilation of the maximal interaction rates of the different experiment is shown in Fig. 9. Collider experiments (MPD, STAR) can cover a broad energy range, but are typically limited in collision rate by the luminosity of the accelerator, which decreases rapidly when running the machine below the maximal energy. The rate capability of fixed-target experiments, on the other hand, is in general not limited by the beam intensity delivered by the machine, but by the properties of the detector and read-out systems. Naturally, the maximal centreof-momentum energy reachable in fixed-target mode is much smaller than in collider experiments, since most of the beam energy goes into the motion of the centre-of-momentum system with respect to the laboratory. Among the existing experiments, the HADES experiment at SIS-18 shows the highest rate capability $(20 \mathrm{kHz})$ for heavy-ion reactions. CEE and BM@N will run at comparable rates, while CBM ambitiously aims at extreme rates, more than two orders of magnitude higher. The J-PARC plans name even higher rate capabilities, but in contrast to CBM, which has recently already performed full-system tests with pre-series detector elements at rates of several $\mathrm{MHz}$, an experimental concept at J-PARC satisfying such challenging aims is still to be demonstrated. On the collider side, is is encouraging to note that at the energy where the STAR BES-II programme starts to seriously suffer from low interaction rates, MPD at NICA will take over, extending the accessible statistics by some two orders of magnitude.

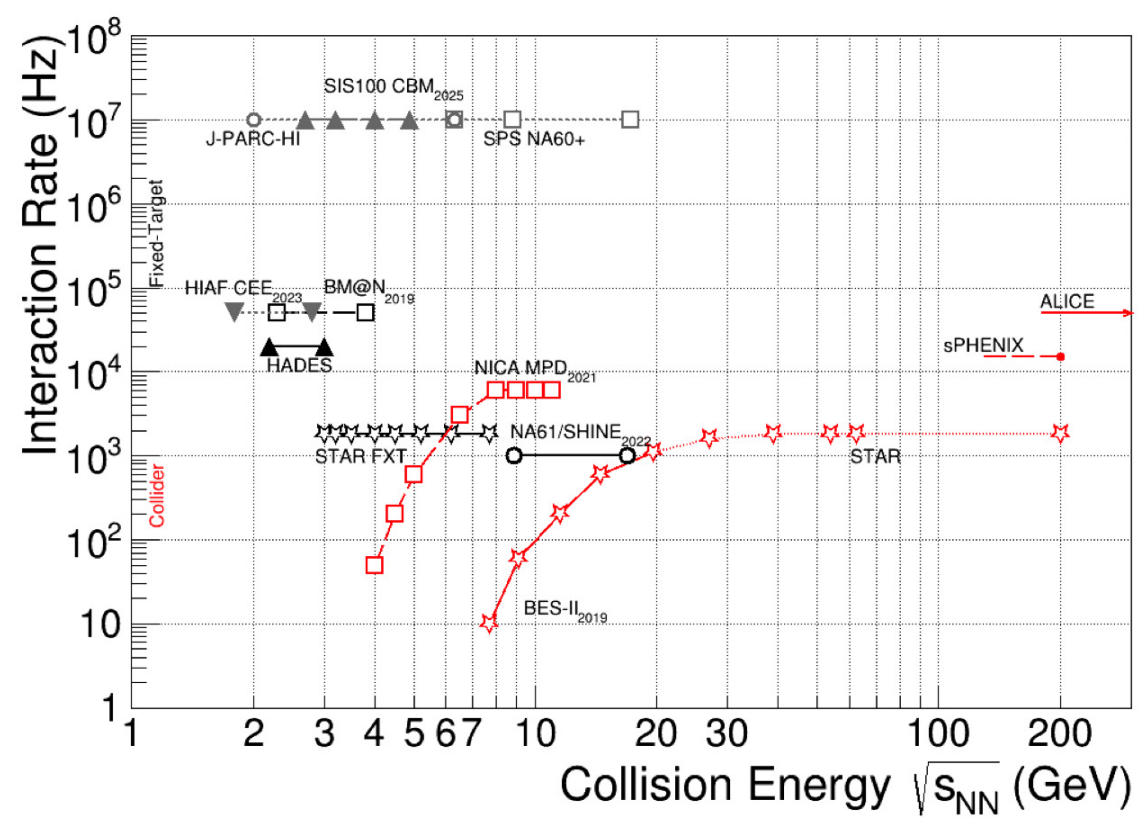

Figure 9: Experimental landscape for the investigation of baryon-rich QCD matter in terms of collision energy range and rate capability $[14,3]$. Existing experiments are denoted by full lines, experiments in preparation by dashed lines, proposed experiments by dotted lines. Collider experiments are shown in red, fixed-target experiments in black or grey.

The physics reach in terms of collected event statistics, however, is not solely determined by the rate capability of the experiment, but also by the availability of beams from the accelerator, i.e., by the allocated beam time. Competition for beams is naturally notorious for experiments with 
extracted beams, while being of less impact in a collider, where several experiments can share the same beam. Here, it is worth noticing that both BM@N and MPD are at the moment the only users of NICA beams, until, at a later stage, the SPD detector will come into operation. In contrast, the FAIR facility will serve a large number of physics programmes, with which CBM will have to compete for beams. CEE at HIAF faces a similar situation. However, the FAIR accelerator concept comprises a large degree of parallel operation of experiments with extracted beams (e.g., CBM) to storage-ring experiments (e.g., PANDA). Out of nine month of FAIR operations per year, CBM expects to run about three months, which appears plenty given the envisaged interaction rates.

For the comparison of the physics capabilities of fixed-target to collider experiments, other aspects are worth being considered. Owing to the kinematical focusing, a fixed-target setup allows to instrument a broad rapidity range up to close to beam rapidity, as can be seen in the setups of BM@N, CBM and CEE, and has no problems measuring down to zero transverse momentum. The barrel-geometry of collider experiments typically covers a symmetric rapidity range around midrapidity. It provides a uniform acceptance in terms of azimuth angle, but does not cover very low transverse momenta. When varying the beam energy, the acceptance stays approximately constant, which is a big advantage e.g. for the measurement of event-by-event fluctuations, since acceptance corrections (and their associated systematic errors) do not depend on the collision energy. In contrast, the detector acceptance w.r.t. the centre-of-momentum of the collision changes with beam energy for fixed-target experiments. This can be partially compensated by varying the magnetic field of the spectrometers, but on the expense of momentum resolution.

The precise determination of event centrality is a pre-requisite in particular for the measurement of event-by-event fluctuations. This task is usually performed by a forward calorimeter. At moderate rates, fixed-target experiments allow to hermetically cover the beam area, thus catching all beam spectator nucleons and beam fragments. However, the target spectators are normally not measured at all, which limits the possibility to select events with a fixed number of participants. In a collider geometry, the spectators an both sides can be measured, but of course, the spectator detectors have to let the beam pass through and consequently also miss the heavier fragments. This is also the case for CBM, although being a fixed-target experiment, since the very high beam intensities forbid to steer the beam into a calorimeter.

\section{Physics perspectives}

\subsection{Hadron production}

The identification of bulk hadrons $(\pi, K, p)$ by means of time-of-flight and/or specific energy loss is a key ingredient of all new experiments discussed above. In addition, all of them provide identification of weak decay topologies by precision tracking devices. Spectra and integrated yields of identified hadrons are required to determine the freeze-out properties of the matter created in the collision, accessible in terms of temperature and baryon-chemical potential obtained by fits to the Statistical Hadron Gas Model. Current data sets at low collision energies suffer from a limited range of hadron species entering the fit. It is thus of importance to extend the menu of hadron species to less abundant ones. Multi-strange hyperons play a key role here, in particular in view of the HADES results on $\Xi^{-}$yields, which differ by a large factor from the expectation of chemical 
equilibrium [22]. BM@N, MPD and CBM data will clarify whether statistical hadronisation also holds for rare baryons at low energies, in particular close to or below the production threshold in elementary collisions.

Multi-strange anti-hyperons are of particular interest, since at high net-baryon densities, their production in a purely hadronic environment is strongly suppressed. They are thus expected to be very sensitive to the nature of the medium, i.e., to an onset of deconfinement or partial restoration of chiral symmetry [23]. Assuming the multiplicities predicted by the purely hadronic UrQMD model [24], MPD will be able to measure $\bar{\Xi}^{+}$at least at the higher energies, while the high rate capability of CBM promises also access to the extremely rare $\bar{\Omega}^{+}$.

\subsection{Hyper-nuclei and exotic strange objects}

In heavy-ion collisions, hyper-nuclei can be produced through the capture of $\Lambda$ baryons by light fragments. The Statistical Hadron Gas Model predicts the maximum of the production yield at low energies $\left(\sqrt{{ }^{S N N}}=4-7 \mathrm{GeV}\right)[25]$ - well suitable for the new experiments discussed in section 2. The experimental techniques - detection of the weak decay vertex and identification of the decay products - are quite similar to those for hyperon measurements and are available for all new experiments. The experimental sensitivity thus depends on statistical arguments only. While singly-strange hyper-nuclei will be accessible by all experiments, the rare doubly-strange species require very high interaction rates. For example, the expected detection yield for ${ }_{\Lambda \Lambda}^{6} \mathrm{He}$ is only 60 per week, even at an event rate of $10^{7} / \mathrm{s}$ [14]. This suggests that CBM, as dedicated high-rate experiment, will have the best chances for such measurements.

The search for exotic objects, such as strange di-baryons or multi-strange short-lived objects, will also be conducted by all new experiments. Models predicts such objects to be created in high-density matter through distillation of strangeness [26]. The experimental sensitivity to these objects will depend on their yet unknown production yield, such that the experimental prospects can hardly be quantified. It is obvious, though, that the discovery potential in this sector depends on the collected event statistics and thus on the rate capability of the respective experiment.

\subsection{Fluctuations}

Event-by-event fluctuations of conserved quantities are an important tool to look for critical behaviour as expected in the vicinity of the QCD critical point, but might also be indicative for a first-order phase transition. The current discussion is focused on the findings of the STAR collaboration of a non-monotonic behaviour of the fourth-order cumulant of the net-proton number $[27,28]$, which was recently complemented by the HADES collaboration at much lower energy [29]. For definite conclusions, it appears important to study the entire energy region between HADES and the lowest STAR energy, and to reduce the substantial statistical and systematic errors. This will be a prime task for the BES-2 programme of STAR, to be conducted in 2019 and 2020.

This measurement requires substantial but not extravagant event statistics, identification of protons, and well understood event centrality determination. All experiments described here come with these properties and can thus be expected to be able to contribute. In view of the fact that corrections of fluctuation observables for finite acceptance and detection efficiency are difficult and not yet fully understood, a uniform and energy-independent acceptance appears advantageous, 
which suggests the MPD as collider experiment to be better suited than the fixed-target experiments. It should be noted, though, that there a quite a number of open questions, e.g., the validity of the approximation of net-baryon number by proton number at lower energies, where a fraction of protons is bound in light fragments. Results from fixed-target experiments will help to clarify these issues and to fully understand the systematic uncertainties. Moreover, at the lowest energies, only the fixed-target experiments (STAR-FT, CEE, BM@N, CBM) will come with the required rate capability.

\subsection{Thermal di-leptons}

Owing to their penetrating nature, lepton pairs are a prime tool to probe the matter produced in heavy-ion collisions at all stages of the fireball evolution. The observables relevant for he study of the deconfinement and chiral transitions and the critical point are the low-mass excess $(M<$ $1 \mathrm{GeV})$, the apparent temperature at intermediate masses $(1 \mathrm{GeV}<M<2.5 \mathrm{GeV})$, the elliptic flow of lepton pairs, and their polarisation. Experimentally, the measurement requires identification of electrons or muons with a high degree of purity. While the access to the low-mass range requires moderate event statistics $\left(\mathrm{O}\left(10^{7}\right)\right)$, a precision measurement of the intermediate-mass range above $1 \mathrm{GeV}$ is very statistics-hungry because of the yield decreasing exponentially with mass.

Both the MVD and the CBM experiment are capable of electron identification; the performance of both detectors for di-electron measurements in terms of signal-to-background ratio is competitive to dedicated lepton-pair experiments (NA60, CERES) [14, 30]. CBM will, in addition, measure muon pairs within the same acceptance. Because the background sources are quite different for electrons and muons, having both measurements within one and the same apparatus is expected to provide a better assessment of the systematic errors for these complicated observables.

\subsection{Charm}

The measurement of charmed hadrons in the energy range discussed here is very challenging owing to the extremely low production multiplicities. Detection of charm decay topologies, with decay vertices typically a few millimeters or even less distant from the main interaction vertex, requires high-precision tracking devices. Most experiments, e.g. STAR, use silicon pixel detectors for this purpose. An upgrade of the NA61 detectors with such devices has recently been achieved and been successfully operated [31]. NA61 intends to further enhance its micro-vertexing capabilities and expects to be sensitive for open charm measurements in the upper SPS energy range (> $60 \mathrm{GeV} / \mathrm{u}$ ) in a run campaign starting 2022.

Among the new experiments, the MPD detector foresees the installation of a Inner Tracking System in the second phase of the experiment, starting 2023; the sensitivity to open charm decays in heavy-ion collisions, however, is not yet proven [32]. The CBM detector also features detection of open charm by a Micro-Vertex Detector (MVD) [14]. The maximum beam energy for heavy ions provided by the SIS-100 accelerator of FAIR, however, is below the D meson production threshold in $\mathrm{p}+\mathrm{p}$ collisions. In addition, the CBM-MVD has a rate capability of $300 \mathrm{kHz}$ only, unlike the rest of the CBM detectors. It is at current not clear whether a sub-threshold measurement of open charm with $\mathrm{Au}+\mathrm{Au}$ collisions will be possible. With symmetric nuclei (e.g., $\mathrm{Ni}+\mathrm{Ni}$ ), $\mathrm{CBM}$ could measure at $14 \mathrm{GeV} / \mathrm{u}$, where detection of $\mathrm{D}$ mesons will be in reach. The CBM potential for open charm 
measurements will be greatly enhanced when beams from the additional booster synchrotron will become available, which, however, cannot be expected in the next decade.

CBM is the only of the approved experiments which aims at the measurement of charmonium. This will be performed in the di-muon decay channel, which does not suffer from the rate limitations of the MVD detector and can thus make use of the maximal rate capability. CBM reports to be sensitive to both sub-threshold $J / \psi$ production in $\mathrm{Au}+\mathrm{Au}$ collisions as well as to the same observable in $\mathrm{Ni}+\mathrm{Ni}$ collisions at $14 \mathrm{GeV} / \mathrm{u}$ [14]. The possibility of a comprehensive study of the energy dependence of charmonium production, however, will open up only with the second synchrotron in FAIR phase-2.

\section{Summary}

The experimental investigation of QCD matter at high net-baryon densities using nuclear collisions at moderate energies continues to be a lively research field. In the next decade, a number of new accelerator facilities and dedicated experiments will come into operation. Each of these experimental projects, as well as the already running experiments, come with pros and cons in terms of energy range, interaction rate, availability, acceptance, menu of observables, and event characterisation. Comparing the experimental approaches, we find a large degree of complementarity, such that from the entirety of efforts we can expect a wealth of data which will allow a substantially improved insight into the nature of the created matter. Provided that theoretical efforts keep pace with the experimental ones, decisive progress will be made in pinning down the key features of the phase diagram of strongly interacting matter: the equation of state of baryon-rich QCD matter, the deconfinement and chiral phase transitions, and the critical point.

\section{Acknowledgments}

We are grateful to T. Galatyuk, M. Kapishin, T. Sakaguchi and N. Xu for providing information and material.

\section{References}

[1] P. Steinbrecher, arXiv:1807.05607 [hep-lat]

[2] B. Friman et al. (Editors), The CBM Physics Book, Lect. Notes Phys. Vol. 814, Heidelberg 2011

[3] T. Galatyuk, Nucl. Phys. A 982 (2019) 163

[4] C. Yang for the STAR collaboration; Nucl. Phys. A 967 (2017) 800

[5] A. Aduszkiewicz et al. (NA61/SHINE collaboration, CERN-SPSC-2018-008, SPSC-P-330-ADD-10

[6] G. Usai, Nucl. Phys. A 931 (2014) 729

[7] http://nica.jinr.ru

[8] V. Kekelidze et al., EPJ Web Conf. 182 (2018) 02063

[9] A. Galavanov et al., EPJ Web Conf. 204 (2019) 07009

[10] Figures courtesy of M. Kapishin 
[11] www.fair-center.eu

[12] Figures courtesy of FAIR GmbH

[13] Photo: D. Fehrens / GSI Helmholtzzentrum für Schwerionenforschung mbH

[14] T. Ablyazimov et al. (CBM Collaboration), Eur. Phys. J. A 53 (2017) 60

[15] P. Senger and N. Herrmann, Nucl. Phys. News 28 (2018) 23

[16] V. Friese et al. (CBM Collaboration), J. Phys. Conf. Ser. 898 (2017) 112003

[17] J. C. Yang et al., Nucl. Instrum. Methods B 317 (2013) 263

[18] http://imp.cas.cn

[19] N. Xu, priv. comm. (2018)

[20] H. Sako et al., JPS Conf. Ser. 8 (2015) 022010

[21] T. Sakaguchi (for the J_PARC_HI collaboration), these proceedings (arXiv:1904.12821 [nucl-ex])

[22] G. Agakishiev et al. (HADES collaboration), Phys. Rev. Lett. 103 (2009) 132301

[23] W. Cassing and E. Bratkovskaya, Nucl. Phys. A 831 (2009) 215. A similar sensitivity to partonic effects was also obtained with the AMPT model, comparing results with and without string melting (CBM collaboration, priv. comm.)

[24] S. Bass et al., Prog. Part. Nucl. Phys. 41 (1998) 225

[25] A. Andronic et al., Phys. Lett. B 697 (2011) 203

[26] H. Stöcker et al., Nucl. Phys. A 827 (2009) 624c

[27] X. Luo (for the STAR collaboration), PoS(CPOD2014) (2014) 019

[28] J. Thäder (for the STAR collaboration), Nucl. Phys. A 956 (2016) 320

[29] M. Szala (for the HADES collaboration), J. Phys.: Conf. Ser. 1024 (2018) 012024

[30] MPD collaboration, Conceptual design report for the MPD experiment, http://mpd.jinr.ru/wp-content/uploads/2016/04/MPD_CDR_en.pdf

[31] D. Larsen and A. Merzlaya (NA61 collaboration), J. Phys.: Conf. Ser. 1137 (2018) 012048

[32] D. Zinchenko, E. Nikonov and A. Zinchenko, Eur. Phys. J. Web Conf. 204 (2019) 07006 